\title{
Relaciones entre las fases del proceso de gestión del conocimiento en unidades académicas
}

Emilio R. Rodríguez-Ponce ${ }^{1}$, Liliana M. Pedraja-Rejas ${ }^{2}$, Carmen A. Araneda-Guirriman ${ }^{2}$ y Camila P. Muñoz-Fritis ${ }^{2}$ (1) Instituto de Alta Investigación, Universidad de Tarapacá, Antofagasta 1520, Arica-Chile. (Correo-e: erodriguez@uta.cl) (2) Ingeniería Industrial y de Sistemas, Universidad de Tarapacá, 18 de septiembre 2222, Arica-Chile

(Correo-e: Ipedraja@uta.cl; caraneda@uta.cl; camila.munoz.fritis@gmail.com)

Recibido Abr. 26, 2021; Aceptado Jun. 22, 2021; Versión final Ago. 28, 2021, Publicado Feb. 2022

\section{Resumen}

La presente investigación tiene como propósito explorar las relaciones existentes entre las diferentes fases de la gestión del conocimiento: crear, almacenar, compartir y aplicar conocimiento. Se realizó un estudio exploratorio con una muestra de 35 líderes de unidades académicas de cinco instituciones chilenas. Se efectuó un análisis econométrico empleando el software SPSS, descubriéndose así la existencia de un conjunto de relaciones estadísticamente significativas entre distintas fases del proceso analizado. En concreto, los hallazgos evidenciaron que la aplicación de conocimiento depende directamente de los subprocesos de crear y compartir conocimiento. A su vez, la fase de almacenar conocimiento depende directamente de la fase de crear conocimiento y es un subproceso significativo para explicar la fase de compartir conocimiento. Se concluye que la validación de las variables utilizadas, su fiabilidad, y la exploración de relaciones con soporte teórico pone en relieve la importancia de promover y cultivar una gestión del conocimiento de alta calidad en cada una de sus fases.

\section{Relationships between knowledge management process phases in academic units}

\begin{abstract}
This research study aimed to explore the relationships between different phases of knowledge management: create, store, share, and apply knowledge. An exploratory study was applied with a sample of 35 academic unit leaders from five Chilean institutions. An econometric analysis was performed using the SPSS software. This revealed statistically significant relationships between different phases of the knowledge management process. The results showed that the application of knowledge depends directly on the sub-processes of creating and sharing knowledge. In addition, storing knowledge depends directly on creating knowledge and is required to explain the sharing phase. It is concluded that validating the variables examined, including their reliability, and exploring their relationships within a theoretical framework highlight the importance of promoting and enhancing knowledge management in each of its phases.
\end{abstract}




\section{INTRODUCCIÓN}

En la sociedad del conocimiento la fuente esencial de la riqueza de los países estriba precisamente en el conocimiento, ya que este es reconocido como un recurso con un gran potencial para generar ventajas competitivas sostenibles al proporcionar un marco para evaluar e incorporar nuevas experiencias e información al quehacer organizacional (Echeverri et al., 2018). Ciertamente, la generación de innovaciones y el mejoramiento de la productividad constituyen resultantes concretas del conocimiento individual y del conocimiento colectivo, los cuales impactan sobre el desarrollo de las organizaciones, como de la sociedad en su conjunto.

En el ámbito de la educación superior, se ha planteado que la gestión del conocimiento juega un rol importante en el éxito de estas instituciones, debido principalmente a que la planificación, organización, monitoreo y coordinación efectiva de los activos de este proceso se relacionan con el capital intelectual (Nair y Munusami, 2019), la innovación, y en consecuencia con el desempeño organizacional (lqbal et al., 2019). Asimismo, se ha señalado que esta es igualmente relevante en las entradas y salidas del proceso educativo, en sentido de que la mejora del conocimiento de los estudiantes se relaciona directamente con el conocimiento que tienen estas instituciones (Al-Jedaiah, 2020). Así las cosas, diversos estudiosos han planteado que las universidades deben adoptar un enfoque estratégico para las prácticas de gestión del conocimiento de manera que se apoye al cumplimiento de su misión (investigación, docencia y servicio a la comunidad) (Veer-Ramjeawon y Rowley, 2020).

Ahora bien, la gestión del conocimiento es el proceso sistemático, permanente e ininterrumpido que, a través de los subprocesos de crear, almacenar, compartir y aplicar conocimiento, permite a las organizaciones crear nuevos productos o servicios y mejorar la capacidad de resolución de problemas, creando ventajas competitivas sustentables (Ferreira et al., 2018). El resultado final del proceso de gestión de este es la aplicación del conocimiento, para lo cual se requieren realizar una serie de subprocesos o fases previas (Niqresh, 2021). En definitiva, la creación de conocimiento es un subproceso esencial en las organizaciones; también lo es su almacenamiento y el subproceso de compartirlo, pero sólo la aplicación de conocimiento efectivo tendrá efectos en los resultados finales (Abubakar et al., 2019). Por lo mismo el estudiar las relaciones entre las diversas fases debería ayudar a comprender en qué subprocesos se deben focalizar los esfuerzos organizativos para lograr una plena aplicación del conocimiento generado en la organización, y, en consecuencia, aprovechar sus múltiples beneficios.

Bajo esta perspectiva, se realiza la presente investigación de tipo exploratoria para identificar las posibles relaciones existentes entre las diferentes fases de la gestión del conocimiento en cinco instituciones chilenas de educación superior. La importancia de este estudio radica en que las investigaciones sobre la gestión del conocimiento en este campo son relativamente recientes (Veer-Ramjeawon y Rowley, 2020), y, por tanto, la idea es contribuir a la literatura con evidencia empírica específica desde un país emergente como lo es Chile, aportando a precisar qué dimensiones de la gestión del conocimiento pueden influir en las fases posteriores, hasta llegar a cristalizar el proceso en la aplicación concreta de conocimientos.

\section{OTROS ANTECEDENTES}

La gestión del conocimiento consiste en un conjunto de subprocesos y actividades que permiten a las organizaciones generar, adquirir, descubrir y organizar conocimiento, posibilitando su uso y difusión en los diferentes niveles de la organización (Hussinki et al., 2017). En línea con la conceptualización anterior y en forma previa, Zhao y Lavín (2012) habían planteado que la gestión del conocimiento es un proceso esencial para las organizaciones, ya que permite la creación de conocimiento inicialmente en forma individual, para luego compartirlo entre los miembros de una organización y aplicarlo tanto para generar innovaciones o mejoras en los productos o servicios, como para la adopción de decisiones e ideas que buscan el mejoramiento de las instituciones. En efecto, el proceso de gestión del conocimiento posibilita que las organizaciones obtengan, organicen, generen y diseminen conocimiento para generar productos y/o servicios. Asimismo, un elemento adicional a tener en consideración es que la gestión del conocimiento favorece la toma de decisiones y los procesos de dirección estratégica en las instituciones (Rodríguez-Ponce y PedrajaRejas, 2016).

En este marco, se puede establecer que la gestión del conocimiento está configurada a partir de una serie de subprocesos que consisten en: crear, almacenar, compartir y aplicar conocimiento (Abualoush et al., 2018). La creación de conocimiento surge de los individuos, quienes a través de su labor diaria exploran y combinan, intuitiva e interactivamente, sus conocimientos creando un nuevo acervo (Canonico et al., 2020). Existen diferentes modos de crear conocimiento, entre las que destacan: la socialización, la externalización, la combinación, y la internalización; las cuales son clave para mejorar la arquitectura de innovación en las organizaciones (Azzam et al., 2020). 
El subproceso de almacenamiento de conocimiento solo es posible una vez que dicho conocimiento haya sido creado, y consiste en diseñar e implementar los sistemas y mecanismos que permitan almacenar, actualizar y recuperar eficientemente el conocimiento generado (Costa y Monteiro, 2018), el cual debe ser accesible y fácil de usar por quienes lo necesiten en la institución. Dicho de modo claro, la eficaz distribución del conocimiento resulta ser una condición esencial para el subproceso de almacenamiento (Kapuza et al., 2020).

A su turno, el proceso de compartir conocimiento emerge una vez que este ya ha sido creado y/o almacenado, es decir, en cuanto existe un nuevo conocimiento y se produce un intercambio que permite la transferencia colectiva del mismo (Appel-Meulenbroek et al., 2018). Como lo señalan Bashir y Farooq (2019), al compartir conocimiento se generan sinergias que hacen que el conocimiento inicial aumente su valor y su potencial de impacto positivo en las organizaciones. Por último, el subproceso de aplicación de conocimiento tiene como propósito la creación de valor estratégico, sea mediante la generación de innovaciones que se traducen en nuevos procesos o productos (Mahdi et al., 2019) o incluso mejorando la capacidad de respuesta y las decisiones corporativas (Hughes y Hodgkinson, 2020). Es por esto que se plantea que aplicar conocimiento es imprescindible para alcanzar altos niveles de desempeño.

Ahora bien, la evidencia empírica ha demostrado que los distintos subprocesos de la gestión del conocimiento pueden estar relacionados entre ellos tanto de manera directa como indirecta. En este contexto, se ha descubierto, por ejemplo, que las fases de crear, compartir y aplicar conocimiento no son independientes entre sí, sino más bien, forman parte de un proceso inclusivo e integral (Rodríguez-Ponce y Pedraja-Rejas, 2016), afirmación que se ve respaldada por otros estudios que plantean que todas las fases del proceso de gestión del conocimiento están significativamente correlacionadas entre sí (Gopinath, 2019; Ramadan et al., 2017). A su turno, se ha hallado que las fases de la gestión del conocimiento, a saber, crear, almacenar e intercambiar conocimiento, se relacionan con la aplicación de este, y que dichas fases (salvo en el caso de la fase de intercambio) poseen un efecto significativo y positivo en la innovación de la organización (Ode y Ayavoo, 2020). Adicionalmente, se ha descubierto que el proceso de almacenamiento y documentación de conocimiento impulsado por la tecnología, así como por la capacidad de absorción realizada por las instituciones refuerza el proceso de creación de conocimiento (Costa y Monteiro, 2018).

Por otra parte, en la literatura se ha hallado igualmente que la creación de conocimiento tiene una correlación positiva y significativa con la aplicación del mismo (Duan et al., 2020), y que la capacidad de absorción de conocimiento tiene un efecto de mediación total entre el intercambio de conocimiento entrante y el desempeño de la innovación organizacional (Zhao et al., 2020). A su vez, existen estudios que plantean que el almacenamiento de conocimiento juega un papel clave en la generación, el intercambio y la utilización de este (Demir et al., 2021). Por lo demás, existe evidencia que el impacto de la generación y el intercambio de conocimientos sobre la aplicación de este está mediado por la captura de conocimientos (Zaim et al., 2019). Mientras que hay autores que plantean que el intercambio de conocimientos se relaciona positivamente con la creatividad del equipo, lo cual está totalmente mediado tanto por la capacidad de absorción como por la integración del conocimiento (Men et al., 2019). Finalmente, existe evidencia empírica en la literatura que sugiere igualmente que la adquisición y creación del conocimiento influyen en la aplicación del mismo (la cual incide a su vez en el desempeño organizacional), y que la adquisición y transferencia del conocimiento influye en la creación de este (Seleim y Khalil, 2007).

Considerando los antecedentes teóricos y empíricos anteriormente expuestos, se cree pertinente suponer que las distintas fases de la gestión del conocimiento se relacionan entre sí, y que los subprocesos de crear, almacenar y compartir conocimiento afectan a las fases posteriores. De este modo, se buscan probar las siguientes hipótesis: H1. El subproceso de almacenar conocimiento se ve afectado por la fase de crear conocimiento; H2. Los subprocesos de crear y almacenar afectan a la fase de compartir conocimiento; y H3. El subproceso de aplicar conocimiento se ve afectado por las fases de crear, almacenar y compartir conocimiento.

\section{METODOLOGÍA}

Dado que las investigaciones sobre la gestión del conocimiento en el campo de la educación superior son relativamente recientes (Veer-Ramjeawon y Rowley, 2020), este artículo se plantea como exploratorio, en sentido de que se busca dar un primer paso en una temática poco estudiada por la academia. La importancia de este tipo de investigaciones es que constituyen la base de investigaciones futuras más concluyentes. En concreto, en este estudio se trabaja a pequeña escala para identificar a través de datos empíricos los aspectos fundamentales que proporcionen una visión general, de tipo aproximativo, de las eventuales relaciones entre los subprocesos que configuran la gestión del conocimiento (crear, almacenar, compartir y aplicar conocimiento). 


\section{Participantes y procedimiento de recolección de datos}

Se aplicó un cuestionario a una muestra de 35 líderes de equipos de dirección intermedia de unidades académicas pertenecientes a cinco entidades de educación superior que llevan a cabo su labor en Chile. Debe indicarse que, si bien los líderes representan a equipos que no forman parte del equipo directivo de la institución, se trata de unidades que adoptan e implementan decisiones relevantes para sus respectivas instituciones. Al ser una investigación exploratoria el criterio de selección de la muestra fue tomado por conveniencia, esto debido a que se consideró la disposición y disponibilidad a colaborar para recabar la información necesaria.

\section{Variables y medidas}

Como se ha señalado en el marco conceptual, la gestión del conocimiento se divide en cuatro variables, a saber, crear conocimiento, almacenar conocimiento, compartir conocimiento y aplicar conocimiento. En este sentido, se empleó el planteamiento conceptual de Abualoush et al. (2018) como base para la construcción del instrumento. Se utilizó una escala Likert de siete puntos para medir los 17 ítems. La Tabla 1 muestra las afirmaciones utilizadas, así como los índices de fiabilidad para cada una de las variables analizadas. Tal como se puede observar en esta, todas las variables obtuvieron un alfa mayor a 0,7 , por lo cual, el instrumento se considera altamente consistente (Taber, 2018).

Tabla 1: Índices de fiabilidad.

\begin{tabular}{|c|c|c|c|}
\hline Variables & Afirmación & $\begin{array}{c}\text { Alfa de } \\
\text { Cronbach }\end{array}$ & Fiabilidad \\
\hline $\begin{array}{l}\text { Crear } \\
\text { conocimiento }\end{array}$ & $\begin{array}{l}\text { El equipo de trabajo tiene un sistema eficiente de exploración de la } \\
\text { información interna y externa. } \\
\text { La información obtenida por diferentes fuentes es integrada al interior del } \\
\text { equipo de trabajo. } \\
\text { El equipo de trabajo tiene un sistema que le permite identificar hallazgos } \\
\text { relevantes tanto de fuentes internas como externas. } \\
\text { El equipo de trabajo crea nuevos conocimientos que favorecen sus acciones y } \\
\text { decisiones basados en su sistema de exploración, detección de hallazgos e } \\
\text { integración de información. } \\
\text { Los miembros del equipo interactúan sistemáticamente para generar nuevos } \\
\text { conocimientos. }\end{array}$ & 0,897 & Muy alta \\
\hline $\begin{array}{l}\text { Almacenar } \\
\text { conocimiento }\end{array}$ & $\begin{array}{l}\text { El equipo de trabajo tiene claros sistemas para almacenar los conocimientos } \\
\text { generados. } \\
\text { El conocimiento almacenado puede ser rápidamente recuperado para acceso } \\
\text { de los miembros del equipo de trabajo. } \\
\text { El equipo de trabajo cuenta con un sistema que permite la disponibilidad y } \\
\text { accesibilidad para una distribución eficaz del conocimiento. } \\
\text { El equipo de trabajo valora el almacenamiento del conocimiento como un } \\
\text { aspecto esencial de su labor. }\end{array}$ & 0,891 & Muy alta \\
\hline $\begin{array}{l}\text { Compartir } \\
\text { conocimiento }\end{array}$ & $\begin{array}{l}\text { El equipo de trabajo intercambia conocimientos en forma eficaz y eficiente. } \\
\text { El equipo de trabajo es capaz de transformar el conocimiento individual en } \\
\text { conocimiento grupal. } \\
\text { El equipo de trabajo se transfiere conocimientos en forma eficaz y eficiente. } \\
\text { El equipo de trabajo comparte conocimientos en forma eficaz y eficiente. }\end{array}$ & 0,780 & Alta \\
\hline $\begin{array}{l}\text { Aplicar } \\
\text { conocimiento }\end{array}$ & $\begin{array}{l}\text { El equipo de trabajo aplica los conocimientos generados y compartidos. } \\
\text { El equipo de trabajo toma decisiones en base a los conocimientos generados } \\
\text { y compartidos en su interior. } \\
\text { El equipo de trabajo basa sus actuaciones en los conocimientos generados y } \\
\text { compartidos en su interior. } \\
\text { El equipo de trabajo emplea sistemáticamente el conocimiento generado y } \\
\text { compartido. }\end{array}$ & 0,778 & Alta \\
\hline
\end{tabular}

\section{Análisis de datos}

Los datos obtenidos mediante el cuestionario fueron procesados en el sistema IBM SPSS versión 27 para su análisis estadístico. Se trabajó con un nivel de significancia del $10 \%$ debido a que se trata de una investigación de naturaleza exploratoria. Las ecuaciones 1,2 y 3 representan los modelos de regresión lineal múltiple analizados. En cada caso se representa a: $\alpha$ como la constante que considera las variables omitidas por el modelo; $\beta i$ como el ponderador de la variable independiente; y $\varepsilon$ i como el término de error. Cabe mencionar que, debido a que se utilizó una escala de Likert de siete puntos, los ítems que respondieran a una misma variable se promediaron y se trabajó, posteriormente, con esos promedios a efectos de analizar los modelos de regresión lineal múltiple presentados anteriormente. 
Almacenar conocimiento $=\alpha+\beta 1$ Crear conocimiento $+\varepsilon i$

Compartir conocimiento $=\alpha+\beta 1$ Crear conocimiento $+\beta 2$ Almacenar conocimiento $+\varepsilon i$

Aplicar conocimiento $=\alpha+\beta 1$ Crear conocimiento $+\beta 2$ Almacenar conocimiento $+\beta 3$ Compartir conocimiento $+\varepsilon i$

\section{RESULTADOS Y DISCUSIÓN}

La Tabla 2 muestra las estadísticas descriptivas obtenidas en el análisis. Al observar la media de las respuestas entregadas por los encuestados, los resultados indican que cada una de las cuatro fases de la gestión del conocimiento dista significativamente de llegar a los niveles máximos o ideales (siete por el tipo de escala utilizada).

Tabla 2: Estadísticos descriptivos.

\begin{tabular}{|l|c|c|c|c|c|}
\hline \multicolumn{1}{|c|}{ Variables } & $N$ & Mínimo & Máximo & Media & Desviación estándar \\
\hline Crear conocimiento & 35 & 2,20 & 6,00 & 4,623 & 0,916 \\
\hline Almacenar conocimiento & 35 & 2,00 & 5,50 & 4,271 & 0,830 \\
\hline Compartir conocimiento & 35 & 3,00 & 7,00 & 4,300 & 0,759 \\
\hline Aplicar conocimiento & 35 & 3,25 & 7,00 & 4,579 & 0,851 \\
\hline
\end{tabular}

Al analizar el primer modelo de regresión lineal (véase ecuación 1), se tiene que el subproceso de crear conocimiento explica el $40,4 \%$ de la varianza del subproceso de almacenar conocimiento, resultados que sugieren una alta significancia estadística (Test $F=24,029 ; p<0,001$ ). El ponderador estandarizado es altamente significativo (Test $t=4,902 ; p<0,001$ ). En cualquier caso, es menester destacar que el modelo omite otras variables relevantes para explicar el almacenamiento del conocimiento (Test t de la constante= 2,749; $\mathrm{p}<0,010)$. La Tabla 3 sintetiza los resultados obtenidos en relación al resumen, el análisis de la varianza (ANOVA) y los coeficientes del modelo.

Tabla 3: Resumen del modelo, ANOVA y coeficientes para la variable almacenar conocimiento.

\begin{tabular}{|l|}
\hline Resumen del modelo \\
\hline Variable dependiente: Almacenar conocimiento \\
Variables predictoras: Crear conocimiento \\
$R^{2}=0,421 ; R^{2}$ corregido= 0,$404 ;$ Error típico de la estimación= 0,$641 ;$ Grados de libertad= 1 y 33; \\
$F=24,029 ;$ significación $F<0,001$ \\
\hline ANOVA \\
\hline Suma de cuadrados $=9,869$ (regresión) y 13,553 (residual) \\
Media cuadrática $=9,869$ (regresión) y 0,411 (residual) \\
\hline Coeficientes \\
\hline $\begin{array}{l}\text { A (constante) }=1,553 \pm 0,565 ; \text { test } t=2,749 ; \text { significación= } 0,010 \\
\text { Crear conocimiento }=0,588 \pm 0,120 ; \text { test } t=4,902 ; \text { significación }<0,001\end{array}$ \\
\hline
\end{tabular}

En relación al segundo modelo analizado (véase ecuación 2), se obtuvo que los subprocesos de crear y almacenar conocimiento explican el $17,8 \%$ de la varianza del subproceso de compartir conocimiento. El almacenamiento del conocimiento es la variable determinante (Test $t=2,506 ; p<0,017$ ) para explicar el subproceso de compartir conocimiento; aunque cabe destacar que existen variables relevantes omitidas en el modelo (Test $t$ de la constante $=3,754 ; p<0,001$ ). La Tabla 4 resume estos resultados.

Al analizar el último modelo (véase ecuación 3), se tiene que los subprocesos de crear, almacenar y compartir conocimiento explican el $23,4 \%$ de la varianza del subproceso de aplicar conocimiento. La creación de conocimiento (test $t=1,823, p<0,078$ ) y el compartir conocimiento (test $t=2,776 ; p<0,009$ ) son las variables determinantes. De todos modos, existen variables relevantes omitidas en el modelo (test $t$ de la constante= $1,889 ; p<0,068)$. La Tabla 5 resume estos hallazgos. La Figura 1 sintetiza las relaciones encontradas en el estudio empírico. 
Tabla 4: Resumen del modelo, ANOVA y coeficientes para la variable compartir conocimiento.

\begin{tabular}{|l|}
\hline Resumen del modelo \\
\hline Variable dependiente: Compartir conocimiento \\
Variables predictoras: Crear conocimiento, almacenar conocimiento \\
$\mathrm{R}^{2}=0,226 ; \mathrm{R}^{2}$ corregido= 0,$178 ;$ Error típico de la estimación= 0,$688 ;$ Grados de libertad= 2 y 32; \\
$\mathrm{F}=4,684 ;$ significación $\mathrm{F}=0,016$ \\
\hline ANOVA \\
\hline Suma de cuadrados $=4,439$ (regresión) y 15,161 (residual) \\
Media cuadrática $=2,219$ (regresión) y 0,474 (residual) \\
\hline Coeficientes \\
\hline $\begin{array}{l}\text { A (constante) }=2,526 \pm 0,673 ; \text { test } t=3,754 ; \text { significación< }<0,001 \\
\text { Crear conocimiento }=-0,049 \pm 0,169 ; \text { test } t=-0,291 ; \text { significación }=0,773 \\
\text { Almacenar conocimiento }=0,469 \pm 0,187 ; \text { test } t=2,506 ; \text { significación }=0,017\end{array}$ \\
\hline
\end{tabular}

Tabla 5: Resumen del modelo, ANOVA y coeficientes para la variable aplicar conocimiento.

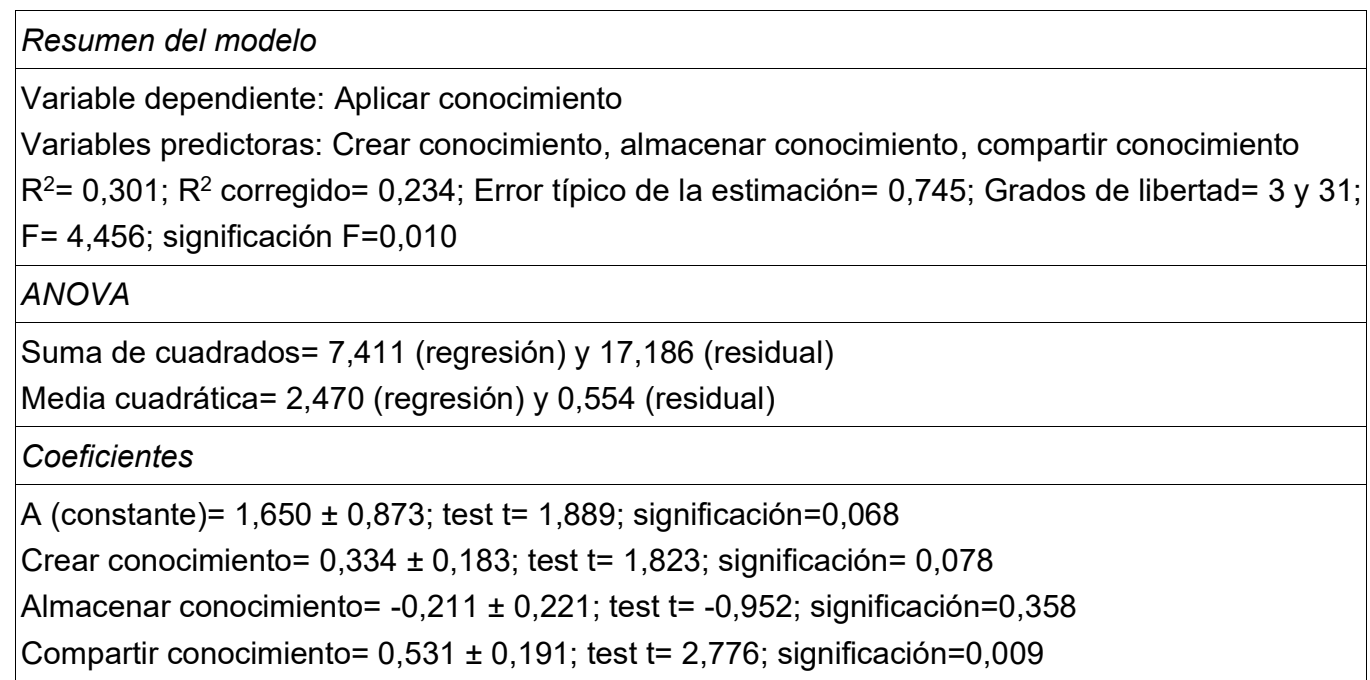

\section{0,334}

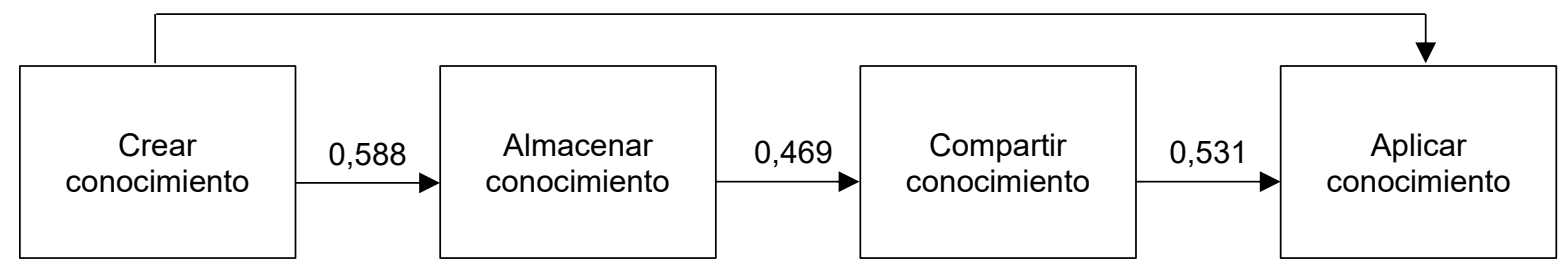

Fig. 1: Relaciones entre las fases de la gestión del conocimiento.

El estudio llevado a cabo tiene como principal limitación su naturaleza exploratoria, lo que significa que los resultados obtenidos no son generalizables estadísticamente. A pesar de esto, el trabajo llevado a cabo contribuye a la literatura sobre gestión de conocimiento, al presentar un estudio empírico realizado en equipos de dirección intermedia, en instituciones de educación superior. En concreto, la investigación proporciona evidencia preliminar a partir de la cual, mediante el análisis de las percepciones de los participantes, se evidenció que las distintas fases de la gestión de conocimiento, a saber, crear, almacenar y compartir conocimiento, tienen efectos directos en los subprocesos que le siguen. Estos hallazgos son consistentes con otros estudios, en relación, a que la gestión del conocimiento es más bien un proceso que integra, y no aísla, cada una de sus fases, las cuales están correlacionadas de manera significativa entre ellas (Gopinath, 2019; Ramadan et al., 2017; RodríguezPonce y Pedraja-Rejas, 2016). Este planteamiento igualmente tiene sustento teórico, en el sentido de que para que se pueda aplicar el conocimiento (resultado final del proceso de gestión del conocimiento), primero se deben realizar una serie de subprocesos o fases previas (Niqresh, 2021), donde la creación, el almacenamiento y el intercambio de conocimiento son esenciales (Abubakar et al., 2019). 
Por otra parte, los hallazgos también evidenciaron que existen otras variables que afectan a las distintas fases de la gestión del conocimiento. Esto de igual manera se encuentra en línea con la literatura, ya que aquí existen estudios empíricos que relacionan a estas con factores relacionados a la cultura de los países (Duan et al., 2020), la capacidad de absorción de las empresas (Costa y Monteiro, 2018; Men et al., 2019), las reuniones intencionales no planificadas y una cultura organizacional de responsabilidad compartida ante los problemas (Appel-Meulenbroek et al., 2018), entre otros.

\section{CONCLUSIONES}

A partir de los resultados obtenidos en este estudio y su posterior discusión se pueden establecer las siguientes cuatro conclusiones relevantes: 1) La gestión del conocimiento es un proceso configurado por una serie de subprocesos (crear, almacenar, compartir y aplicar el conocimiento) que buscan generar valor estratégico para las organizaciones, ya sea mediante la creación de nuevos productos o servicios, o mejorando los procesos estratégicos y sus resultados. Ahora bien, lograr altos niveles de calidad en cada subproceso de la gestión del conocimiento parece evidente, sin embargo, en los hechos reales no lo es. Nótese que los promedios obtenidos en los subprocesos de crear, almacenar, compartir y aplicar conocimiento distan significativamente de los valores ideales; 2) el estudio realizado es de naturaleza exploratoria por lo cual su alcance es limitado. No obstante, la investigación es relevante, ya que proporciona soporte empírico a un conjunto de relaciones que conceptualmente son consideradas pertinentes; 3) en efecto, los hallazgos muestran que la aplicación de conocimiento depende directamente de los subprocesos de crear y compartir conocimiento y, a su vez, almacenar conocimiento es significativo para explicar el subproceso de compartir conocimiento; de igual manera los resultados evidenciaron que almacenar conocimiento depende directamente de crear conocimiento; por último, 4) la validación de las variables utilizadas, su fiabilidad, y la exploración de relaciones con soporte teórico dan cuenta de una aportación que pone en relieve la importancia de promover y cultivar una gestión del conocimiento de alta calidad en cada una de sus fases.

\section{AGRADECIMIENTOS}

Los autores agradecen el patrocinio recibido por la Agencia Nacional de Investigación y Desarrollo de Chile (ANID) a través del proyecto Fondecyt Regular 1180484.

\section{REFERENCIAS}

Abualoush, S., Masa'deh, R., Bataineh, K., y Alrowwad, A., The role of knowledge management process and intellectual capital as intermediary variables between knowledge management infrastructure and organization performance, https://doi.org/10.28945/4088, Interdisciplinary Journal of Information, Knowledge, and Management, 13, 279-309 (2018)

Abubakar, A. M., Elrehail, H., Alatailat, M. A., y Elçi, A., Knowledge management, decision-making style and organizational performance, https://doi.org/10.1016/j.jik.2017.07.003, Journal of Innovation \& Knowledge, 4(2), 104-114 (2019)

Al-Jedaiah, M., Knowledge management and e-learning effectiveness: empirical evidence from Jordanian higher education institutions, https://doi.org/10.3991/ijet.v15i05.11653, International Journal of Emerging Technologies in Learning (iJET), 15(5), 50-62 (2020)

Appel-Meulenbroek, R., Weggeman, M., y Torkkeli, M., Knowledge sharing behaviour within organizations; a diary-based study of unplanned meetings between researchers, https://doi.org/10.1080/14778238.2018.1459254, Knowledge Management Research \& Practice, 16(2), 267-279 (2018)

Azzam, A. A., He, Q., y Sarpong, D., The role of knowledge creation modes in architectural innovation, https://doi.org/10.1002/jsc.2312, Strategic Change, 29(1), 77-87 (2020)

Bashir, M., y Farooq, R., The synergetic effect of knowledge management and business model innovation on firm competence: a systematic review, https://doi.org/10.1108/IJIS-10-2018-0103, International Journal of Innovation Science, 11(3), 362-387 (2019)

Canonico, P., De Nito, E., y otros tres autores, Knowledge creation in the automotive industry: analyzing obeya-oriented practices using the SECl model, https://doi.org/10.1016/j.jbusres.2019.11.047, Journal of Business Research, 112, 450457 (2020)

Costa, V., y Monteiro, S., From potential absorptive capacity to knowledge creation in organizations: the mediating role of knowledge storage and realized absorptive capacity, https://doi.org/10.1142/S0219649218500065, Journal of Information \& Knowledge Management, 17(1), 1-16 (2018)

Demir, A., Budur, T., Omer, H. M., y Heshmati A., Links between knowledge management and organizational sustainability: does the ISO 9001 certification have an effect?, https://doi.org/10.1080/14778238.2020.1860663, Knowledge Management Research \& Practice, 1-14 (2021) 
Duan, Y., Huang, L., y otros tres autores, The moderating effect of cultural distance on the cross-border knowledge management and innovation quality of multinational corporations, https://doi.org/10.1108/JKM-11-2019-0656, Journal of Knowledge Management, 25(1), 85-116 (2020)

Echeverri, A., Lozada, N., y Arias, J. E., Incidencia de las prácticas de gestión del conocimiento sobre la creatividad organizacional, https://doi.org/10.4067/S0718-07642018000100071, Información Tecnológica, 29(1), 71-82 (2018)

Ferreira, J., Mueller, J., y Papa, A., Strategic knowledge management: theory, practice and future challenges, https://doi.org/10.1108/JKM-07-2018-0461, Journal of Knowledge Management, 24(2), 121-126 (2018)

Gopinath, R., Impact of knowledge management practices on organizational effectiveness of self-financing engineering colleges' faculties, International Journal of Scientific Research and Review, ISSN: 2279-543X, 8(5), 32-39 (2019)

Hughes, P., y Hodgkinson, I., Knowledge management activities and strategic planning capability development, https://doi.org/10.1108/EBR-03-2019-0034, European Business Review, 33(2), 238-254 (2020)

Hussinki, H., Ritala, P., Vanhala, M., y Kianto, A., Intellectual capital, knowledge management practices and firm performance, https://doi.org/10.1108/JIC-11-2016-0116, Journal of Intellectual Capital, 18(4), 904-922 (2017)

Iqbal, A., Latif, F., y otros tres autores, From knowledge management to organizational performance: modelling the mediating role of innovation and intellectual capital in higher education, https://doi.org/10.1108/JEIM-04-2018-0083 Journal of Enterprise Information Management, 32(1), 36-59 (2019)

Kapuza, A., Koponen, I. T., y Tyumeneva, Y., The network approach to assess the structure of knowledge: storage, distribution and retrieval as three measures in analysing concept maps, https://doi.org/10.1111/bjet.12938, British Journal of Educational Technology, 51(6), 2574-2591 (2020)

Mahdi, O. R., Nassar, I. A., y Almsafir, M. K., Knowledge management processes and sustainable competitive advantage: an empirical examination in private universities, https://doi.org/10.1016/j.jbusres.2018.02.013, Journal of Business Research, 94, 320-334 (2019)

Men, C., Fong, P., y otros tres autores, When and how knowledge sharing benefits team creativity: the importance of cognitive team diversity, https://doi.org/10.1017/jmo.2017.47, Journal of Management \& Organization, 25(6), 807-824 (2019)

Nair, B. V., y Munusami, C., Knowledge management practices: an exploratory study at the Malaysian higher education institutions, https://doi.org/10.1108/JRIT-01-2019-0008, Journal of Research in Innovative Teaching \& Learning, 13, 174$190(2019)$

Niqresh, M., The reality of applying knowledge management practices and its impact on achieving the quality of higher education in Jordan, https://doi.org/10.36941/jesr-2021-0002, Journal of Educational and Social Research, 11(1), 9-22 (2021)

Ode, E., y Ayavoo, R., The mediating role of knowledge application in the relationship between knowledge management practices and firm innovation, https://doi.org/10.1016/j.jik.2019.08.002, Journal of Innovation \& Knowledge, 5(3), 210-218 (2020)

Ramadan, B. M., Dahiyat, S. E., Bontis, N., y Al-dalahmeh, M. A., Intellectual capital, knowledge management and social capital within the ICT sector in Jordan, https://doi.org/10.1108/jic-06-2016-0067, Journal of Intellectual Capital, 18(2), 437-462 (2017)

Rodríguez-Ponce, E., y Pedraja-Rejas, L., Percepciones sobre la gestión del conocimiento de directivos universitarios de cuatro universidades chilenas, https://doi.org/10.4067/S0718-50062016000400006, Formación Universitaria, 9(4), 41-52 (2016)

Seleim, A., y Khalil, O., Knowledge management and organizational performance in the Egyptian software firms, https://doi.org/10.4018/ijkm.2007100103, International Journal of Knowledge Management, 3(4), 37-66 (2007)

Taber, K. S., The use of Cronbach's alpha when developing and reporting research instruments in science education, https://doi.org/10.1007/s11165-016-9602-2, Research in Science Education, 48(6), 1273-1296 (2018)

Veer-Ramjeawon, P., y Rowley, J., Embedding knowledge management in higher education institutions (HEls): a comparison between two countries, https://doi.org/10.1080/03075079.2019.1608431, Studies in Higher Education, 45(11), 2324-2340 (2020)

Zaim, H., Muhammed, S., y Tarim, M., Relationship between knowledge management processes and performance: critical role of knowledge utilization in organizations, https://doi.org/10.1080/14778238.2018.1538669, Knowledge Management Research \& Practice, 17(1), 24-38 (2019)

Zhao, S., Jiang, Y., Peng, X., y Hong, J., Knowledge sharing direction and innovation performance in organizations https://doi.org/10.1108/EJIM-09-2019-0244, European Journal of Innovation Management, 1-24 (2020)

Zhao, Y., y Lavin, M., An empirical study of knowledge transfer in working relationships with suppliers in new product development, https://doi.org/10.1142/S1363919612003782, International Journal of Innovation Management, 16(2), 1-26 (2012) 